\title{
LA INVESTIGACIÓN DE PREGRADO EN LA ESCUELA SUPERIOR POLITÉCNICA DE CHIMBORAZO: MAPEO SISTEMÁTICO Y ANALÍTICAS.
}

\section{UNDERGRADUATE RESEARCH AT ESCUELA SUPERIOR POLITECNICA DE CHIMBORAZO: SYSTEMATIC MAPPING AND ANALYTICS.}

\begin{abstract}
Rubén Antonio Pazmiño Maji, Mgs.
Magíster en Informática Educativa (Ecuador). Docente de la Escuela Superior Politécnica de Chimborazo Riobamba, Ecuador. rpazmino@espoch.edu.ec
\end{abstract}

Carmen Elizabeth Solis Benavides, Mgs. Magíster en Docencia universitaria e Investigación Educativa (Ecuador). Docente de la Unidad Educativa Combatientes de Tapi, Riobamba, Ecuador. carmene.solis@educacion.gob.ec

Francisco José García Peñalvo, Ph.D. Doctor en Informática (España). Docente del Departamento de Ciencias de la Computación de la Universidad de Salamanca, España. fgarcia@usal.es

Miguel Ángel Conde González, Ph.D. Doctor en formación en la Sociedad del Conocimiento (España). Docente de la Universidad de León, España. miguel.conde@unileon.es

\section{ARTíCULO DE REVISIÓN BIBLIOGRÁFICA}

Recibido: 10 de septiembre de 2018.

Aceptado: 27 de noviembre de 2018.

\section{RESUMEN}

Desde el año 2014 hasta agosto del 2018, Scopus muestra que existen 2180 artículos científicos sobre las Analíticas de Aprendizaje; esto evidencia la importancia y el interés en este tema. La metodología utilizada es el Mapeo Sistemático de literatura que permite responder a preguntas de investigación mediante la búsqueda secuencial, exhaustiva, 
sistemática y auditable de literatura científica. Esta investigación tiene el objetivo de responder a cuatro preguntas importantes en el ámbito de la investigación de pregrado, habiéndose aplicado el Mapeo Sistemático y las Analíticas Descriptivas y Predictivas a los Trabajos de Titulación de la Escuela Superior Politécnica de Chimborazo. El estudio se realizó desde el 2010 hasta el 2017 (8 años) y se utilizaron como fuentes de información el buscador especializado Google Académico, La Red CEDIA y el repositorio DSpace. En la búsqueda inicial se encontraron un total de 7310 Trabajos de Titulación; luego de la aplicación de los criterios de inclusión, exclusión y calidad se analizaron 7097. Este trabajo muestra el aporte de la utilización del Mapeo Sistemático en Trabajos de Titulación de grado, además muestra dos formas de aplicar las Analíticas de Aprendizaje en la investigación y no exclusivamente en el aprendizaje.

Palabras clave: mapeo sistemático, investigación de pregrado, trabajos de titulación, analíticas, Escuela Superior Politécnica de Chimborazo.

\section{ABSTRACT}

From 2014 to August 2018, Scopus shows that there are 2180 scientific articles on Learning Analytics; this shows the importance and interest in this topic. The methodology used is the Systematic Mapping of Literature that answers research questions through sequential, exhaustive, systematic and auditable search of scientific literature. This research aims to answer four important questions in the field of undergraduate research, having applied Systematic Mapping and Descriptive and Predictive Analyzes in the Degree Projects of the Escuela Superior Politécnica de Chimborazo. The study was conducted from 2010 to 2017 (8 years) and Google Scholar, Red CEDIA and DSpace repository were used as sources of information. A total of 7310 Undergraduate Works were found at the initial search; 7097 were analyzed after the application of inclusion, exclusion and quality criteria. This work shows the contribution of the use of Systematic Mapping in Degree Projects as well as two ways of applying Learning Analytics in research and not exclusively in learning.

Keywords: systematic Mapping, undergraduate research, degree projects, analytics, Escuela Superior Politécnica de Chimborazo.

\section{INTRODUCCIÓN}

La Escuela Superior Politécnica de Chimborazo (ESPOCH) es una institución de Educación Superior ecuatoriana que tiene su origen en el Instituto tecnológico Superior de Chimborazo, 
creado mediante Ley No.69,09, expedida por el Congreso Nacional, el 18 de abril de 1969, inicia sus actividades académicas el 2 de mayo de 1972 (espoch, 2018). La Escuela Superior Politécnica de Chimborazo actualmente está conformada por 7 facultades, 26 escuelas y 40 carreras: la Facultad de Informática y Electrónica ("Facultad de Informática y Electrónica Escuela Superior Politécnica de Chimborazo," 2018:28:23) con cuatro escuelas (Diseño Gráfico; Electrónica, Control y Redes Industriales, Electrónica, Telecomunicaciones y Redes; Sistemas) y siete carreras (Diseño Gráfico; Ingeniería en Electrónica, control y redes Industriales; Electrónica y Automatización; Ingeniería en Electrónica, Telecomunicaciones y Redes; Telecomunicaciones; Ingeniería en Sistemas; Software), Facultad de Ciencias Pecuarias ("Facultad de Ciencias Pecuarias - Escuela Superior Politécnica de Chimborazo," 2018:32:28) con dos escuelas (Zootecnia; Industrias Pecuarias) y dos carreras (Ingeniería Zootécnica; Ingeniería en Industrias Pecuarias), la Facultad de Mecánica ("Facultad de Mecánica - Escuela Superior Politécnica de Chimborazo," 2018:35:35) con cuatro escuelas (Mantenimiento; Mecánica; Industrial; Automotriz) y cuatro carreras (Ingeniería en Mantenimiento; Ingeniería Mecánica; Ingeniería Industrial; Ingeniería Automotriz), la Facultad de Recursos Naturales ("Facultad de Recursos Naturales - Escuela Superior Politécnica de Chimborazo," 2018:38:44) con tres escuelas (Agronomía; Ecoturismo; Forestal) y cinco carreras (Agronomía; Turismo Sostenible; Ecoturismo; Ingeniería Forestal; Ingeniería Recursos Naturales Renovables), la Facultad de Salud Pública ("Facultad de Salud Pública Escuela Superior Politécnica de Chimborazo," 2018:44:46) con 4 escuelas (Promoción y Cuidados de la Salud; Nutrición y Dietética; Medicina; Gastronomía) y cuatro carreras (Promoción y Cuidados de la Salud; Nutrición y Dietética; Medicina; Gastronomía), la Facultad de Ciencias ("Facultad de Ciencias - Escuela Superior Politécnica de Chimborazo," 2018:46:41) con cuatro escuelas (Ingeniería Química; Física y Matemática; Ciencias Químicas; Bioquímica Y Farmacia) y diez carreras (Ingeniería Química; Estadística Informática, Matemática; Física; Biofísica; Estadística; Educación Ambiental; Química; Biotecnología Ambiental; Bioquímico Y Farmacia) y la Facultad de Administración de Empresas ("Facultad de Administración de Empresas - Escuela Superior Politécnica de Chimborazo," 2018:19:07) con cinco escuelas (Empresas; Comercio Exterior; Marketing; Contabilidad y Auditoría; Gestión de Transporte) y ocho carreras (Ingeniería En Empresas; Ingeniería en Comercio Exterior; Ingeniería Financiera; Ingeniería en Marketing; Licenciatura en Mercadotecnia; Ingeniería En Contabilidad y Auditoría; Ingeniería en Gestión de Transporte; Licenciatura en Gestión De Transporte).

El Instituto de investigaciones (IDI) es el órgano institucional (IDI, 2014b) que se encarga de fomentar y sistematizar la investigación (IDI, 2018), desde el año 2014 tiene definidas 8 líneas 
y 33 programas de investigación (IDI, 2014a) basados en la clasificación de las áreas del conocimiento de la UNESCO ("UNESCO. General Conference; 36th; Revisión de la Clasificación Internacional Normalizada de la Educación (CINE); 2011,"; UNESCO, 2018:30:04), los Objetivos del Milenio y el Plan del Buen Vivir ("Objetivos-del-Milenio-Balance2014"). Las Comisiones de Investigación y Transferencia de Ciencia y Tecnología de cada Facultad son las encargadas de definir las sub-líneas de investigación de su respectiva Facultad en concordancia con las líneas y programas institucionales y las Comisiones de Titulación de cada Carrera se encargan de que los Trabajos de Titulación guarden relación con estas sub-líneas. La investigación que se realiza actualmente en las universidades ecuatorianas busca transformarse en uno de los principales puntales de la innovación de la economía que tiene actualmente el Ecuador (LOES, 2010; SENPLADES, 2013) ("EI Plan Nacional para el Buen Vivir ya está disponible para la ciudadanía | Secretaría Nacional de Planificación y Desarrollo," n.d.) (“La Ley Orgánica Reformatoria a Ley Orgánica de Educación Superior fue publica en el Registro Oficial - Ediciones Legales", s/f). El reto constituye entonces contribuir a mejorar las condiciones de vida de la población mediante la investigación (Manosalvas, 05/19/; Ramírez, 2010). Los requerimientos para el proceso de titulación de todas las escuelas de la ESPOCH se encuentran especificadas en el REGLAMENTO DE RÉGIMEN ACADÉMICO INSTITUCIONAL, Sección II: REQUERIMIENTOS Y PROCESO DE TITULACION APROBADO MEDIANTE RESOLUCIÓN 126. CP. 2014, REFORMADO MEDIANTE RESOLUCIONES 434.CP.2014 Y 450.CP.2017), artículo 88. De las opciones de titulación. - que indica:

Conforme lo dispuesto por el Reglamento de Régimen Académico (nacional), se estipulan dos opciones de titulación que permiten la validación académica de los conocimientos, habilidades y desempeños adquiridos en la carrera para la resolución de problemas, dilemas o desafíos de una profesión: A. El desarrollo de un trabajo de titulación, basado en procesos de investigación; o, B. La preparación y aprobación de un examen de grado que deberá ser de carácter complexivo articulado al perfil de egreso de la carrera. Ya sea mediante el trabajo de titulación o el examen de grado el estudiante deberá demostrar el manejo integral de los conocimientos adquiridos a lo largo de su formación profesional; el resultado de su evaluación será registrado cuando se haya completado la totalidad de horas establecidas en el currículo de la carrera, incluidas la unidad de titulación y las prácticas preprofesionales. Cada carrera podrá incluir la defensa oral de los Trabajos de Titulación. ** 
** (Artículo agregado mediante resolución 450.CP.2017)" ("Index of /images/Comunicacion/OCTUBRE 2017/COMUNICADOS," 2018:26:09)

Las Analíticas de Aprendizaje impulsan la recopilación y análisis de datos para mejorar el éxito en el aprendizaje de los alumnos (Deitz-Uhler, B. \& Hurn, J.), usando datos adecuados, puede usarse para realizar evaluaciones formativas e iniciar un proceso posterior de toma de decisiones para la mejora (Pazmiño-Maji, Francisco J. García-Peñalvo, Miguel A. CondeGonzález, Marlene J. García-Veloz, \& Johana K. Montoya-Lunavictoria, 2018) y la predicción (Pazmiño-Maji, Francisco J. García-Peñalvo, Miguel A. Conde-González, Paulina E.ValverdeAguirre, \& Johana K. Montoya-Lunavictoria, 2018) del aprendizaje. Las Analíticas de Aprendizaje admiten la detección de anomalías en tiempo real y, por lo tanto, permite a los docentes tomar decisiones orientadas a ayudar con la mejor adquisición de competencias individuales (Fidalgo-Blanco, Sein-Echaluce, García-Peñalvo, \& Conde, 2015). Las Analíticas del Aprendizaje han surgido como un campo de investigación que con los grandes conjuntos de información de la actividad del alumno puede ser utilizada para entender y mejorar el aprendizaje en línea en particular y el aprendizaje en general (Gelan et al., 2018). En esta comunicación, consideraremos la definición dada en la primera Conferencia Internacional sobre las Analíticas de Aprendizaje y conocimiento ("1st International Conference on Learning Analytics and Knowledge 2011 | Connecting the technical, pedagogical, and social dimensions of learning analytics," 2018:54:26) y tomada por la sociedad de investigación en LA: "Learning Analytics es la medición, recopilación, análisis y reportes de los datos sobre los alumnos y sus contextos, con el fin de comprender y optimizar el aprendizaje y los entornos en los que se produce" (Drupal, 2011).

Es importante notar que cada vez se incrementan nuevas técnicas de análisis más óptimas (Pazmiño, García, \& Conde, 2017a) (Naranjo, Rubén, Conde, \& Peñalvo, 2018) a las ya existentes en las Analíticas de Aprendizaje, esto debido a la compatibilidad (Pazmiño, García, \& Conde, 2016) con otras herramientas tales como el Análisis Estadístico Implicativo (Pazmiño, García, Coutrier, \& Conde, 2015) que tuvo su origen en la didáctica de las matemáticas (Pazmiño, 2014).

\section{REVISIÓN TEÓRICA}

\subsection{Las Analíticas y las Analíticas de Aprendizaje}

Las Analíticas de Aprendizaje hacen parte de las Analíticas en general y de las Analíticas Educativas en particular, en esta investigación utilizamos los tipos de Analíticas de 
Aprendizaje aplicables en la Educación Superior como guía para responder las preguntas de investigación planteadas en el Mapeo Sistemático. Los resultados obtenidos se enmarcan en mayor medida y serán de especial beneficio en las Analíticas Académicas debido a que van dirigidos más hacia los gestores y tomadores de decisiones y no a los estudiantes.

Las Analíticas Descriptivas junto con las Analíticas de diagnóstico, las Analíticas de predicción y las Analíticas de prescripción como muestra la Figura 1, forman parte de las cuatro formas de aplicar las Analíticas de Aprendizaje en la Educación Superior (Gartner, 2018, pp. 36-38).

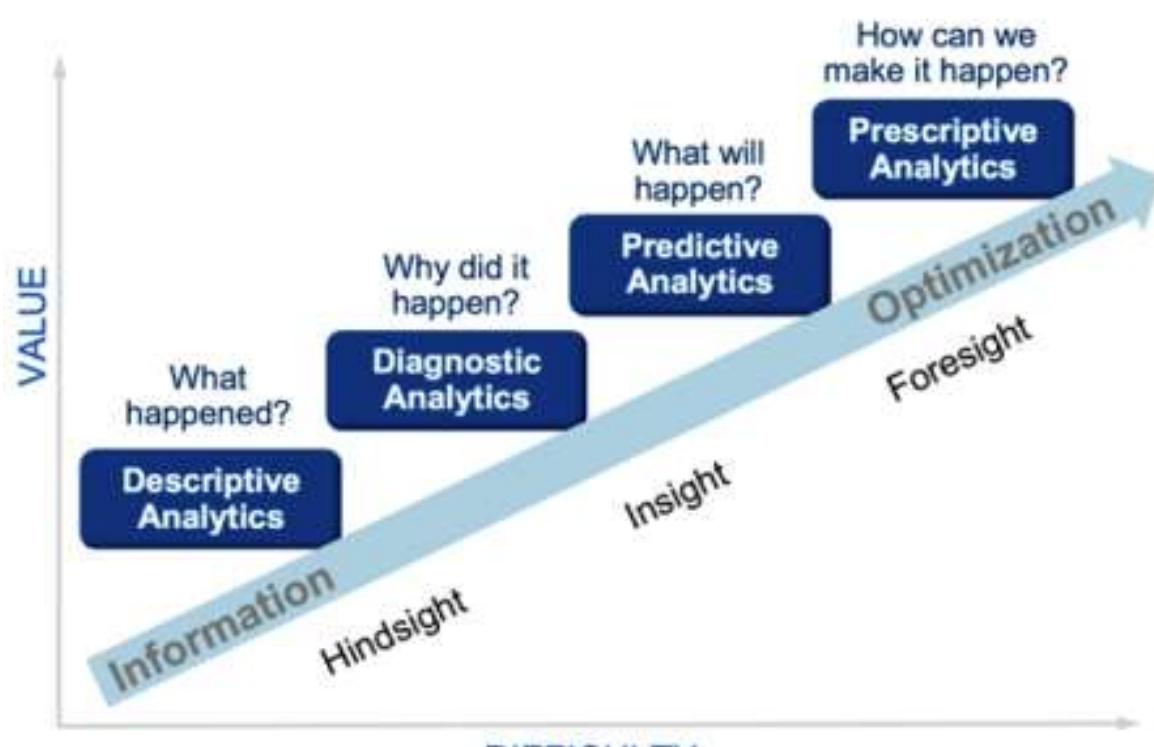

DIFFICULTY

Figura 1. Formas de aplicar las Analíticas de Aprendizaje en la Educación Superior Fuente: Gartner, 2018, pp. 36-38

Las Analíticas Descriptivas responden a la pregunta "¿qué pasó?". Este análisis se puede utilizar en la Educación Superior, por ejemplo, para que el estudiante determine sus avances en el rendimiento académico, su cantidad y calidad de trabajo, su compromiso con los trabajos colaborativos y cooperativos o en el caso de la Investigación sus avances en el desarrollo de los Trabajos de Titulación, la relación de su trabajo de titulación con otros de su misma escuela y carrera, su proximidad a las líneas y programas de investigación institucionales y de carrera (Pazmiño-Maji, Román, Martínez, \& Páez, 2018). Las Analíticas Descriptivas son aplicadas a datos o contenido digital variado como los Trabajos de Titulación, que utiliza técnicas informáticas y estadísticas (como medidas descriptivas o tablas de frecuencia) y visualizaciones (como gráficos de pastel, reloj, barras o líneas), que le permiten tener una idea realista de su proceso de aprendizaje, enseñanza, gestión o investigación. Las Analíticas de Diagnóstico responden a la pregunta ¿por qué sucedió?, busca explicar la situación actual, en la mayoría de los casos se basa en técnicas para el descubrimiento de datos, la minería 
de patrones (Haro, Pazmiño, Conde, \& Peñalvo, 2018) o las correlaciones estadísticas lineales y no lineales. Además, permite comprender qué acciones, sucesos, hechos o su combinación conducen a la situación actualmente detectada. Tales análisis pueden ser utilizados por estudiantes y docentes para descubrir la razón de sus éxitos o fracasos escolares; en el caso de la investigación podría determinar las razones de los avances o retrasos en la ejecución de sus Trabajos de Titulación, las razones por las que determinadas investigaciones se alejan de las líneas de investigación definidas a priori (Pazmiño-Maji, Francisco Peñalvo, Miguel Conde, Paola Chiluiza, \& Diana Campoverde, 2017); o por parte de la institución para evaluar los impactos de acciones específicas para promover la investigación estudiantil, etc. Las Analíticas de Predicción, responden a la pregunta ¿qué pasará?, tiene como objetivo anticipar un futuro cercano basado en la explotación de hechos pasados. Debe poner énfasis en medir la relevancia o confianza de los hechos proyectados y debe ser de fácil interpretación por estudiantes, educadores, investigadores, directivos o responsables de la toma de decisiones. En esta comunicación utilizaremos herramientas accesibles para una gran variedad de usuarios, desde expertos hasta usuarios principiantes.

Las Analíticas de Predicción, son herramientas prometedoras para todos los integrantes del proceso de aprendizaje; permiten por ejemplo a los estudiantes conocer si están trabajando correctamente para lograr sus objetivos de aprendizaje. Al investigador le permite determinar la duración de los recursos utilizados en sus investigaciones, y concordar sus tiempos de trabajo; algunas de las herramientas de análisis frecuentemente utilizadas son el método de promedios móviles de tres períodos. Las Analíticas de Prescripción responden a la pregunta ¿Cómo podemos hacer que suceda?, son otra forma de análisis avanzado que examina datos o contenido digital, para determinar acciones realistas y eficientes, que nos permiten planificar el logro de un objetivo determinado. Se caracteriza por herramientas capaces de procesar gráficos, tratamiento de texto y datos, simulación o extracción de conocimiento (Pazmiño, García, \& Conde, 2017c). Consiste en la obtención de acciones futuras que se deben realizar para lograr un objetivo específico. Este tipo de análisis se puede proporcionar a los estudiantes, para especificar una ruta de aprendizaje o investigación personalizada; a un profesor para sugerir actividades de aprendizaje a sus alumnos; a la Institución de Educación Superior para identificar tendencias en la investigación. En esta comunicación utilizaremos dos formas de aplicar las Analíticas de Aprendizaje en la Educación Superior: las Analíticas Descriptivas y Predictivas, y con ellas responderemos a las preguntas de investigación propuestas en el Mapeo Sistemático de Literatura. 


\subsection{El Mapeo Sistemático de Literatura}

EI Mapeo Sistemático de Literatura es una búsqueda metódica, exhaustiva y auditable de literatura científica en bases de datos bibliográficas especializadas que permite responder a preguntas de investigación generales sobre un tema específico (Petticrew \& Roberts, 2008). Esta investigación tiene el objetivo de responder a 5 preguntas importantes en el ámbito de la investigación de pregrado, para ello se aplicó el Mapeo Sistemático y las Analíticas Descriptivas y Predictivas a 7097 Trabajos de Titulación de la Escuela Superior Politécnica de Chimborazo. Este trabajo además aporta con la utilización del Mapeo Sistemático en Trabajos de Titulación y no únicamente en artículos científicos (Pazmiño, García, \& Conde, 2017b), además muestra dos formas de aplicar las Analíticas de Aprendizaje en la investigación y no exclusivamente en el aprendizaje. En la sección 2 se presentan los pasos de la planificación del Mapeo Sistemático de Literatura. En la sección 3 se pueden observar los resultados y su discusión y en la sección 4 se proponen las conclusiones.

\section{MATERIALES Y METODOS}

Los materiales utilizados fueron las bases de datos bibliográficas Google Académico, La Red CEDIA, el repositorio digital DSpace, el gestor bibliográfico EndNote versión 8.0 y la hoja de cálculo de Microsoft y de Google Docs. La Investigación fue de tipo cuantitativa, no experimental, longitudinal y descriptiva. Se utilizó las Analíticas como fuente de análisis y el Mapeo Sistemático de Literatura (Petticrew \& Roberts, 2008) como fuente secundaria de información y, para su ejecución primeramente se elaboró un protocolo que permitió trabajar con objetividad y hacer la investigación auditable (Okoli \& Schabram, 2010). Los pasos del protocolo utilizado que permitieron ejecutar el Mapeo Sistemático de Literatura se muestran en la Figura 2 y se desarrollan a continuación. 


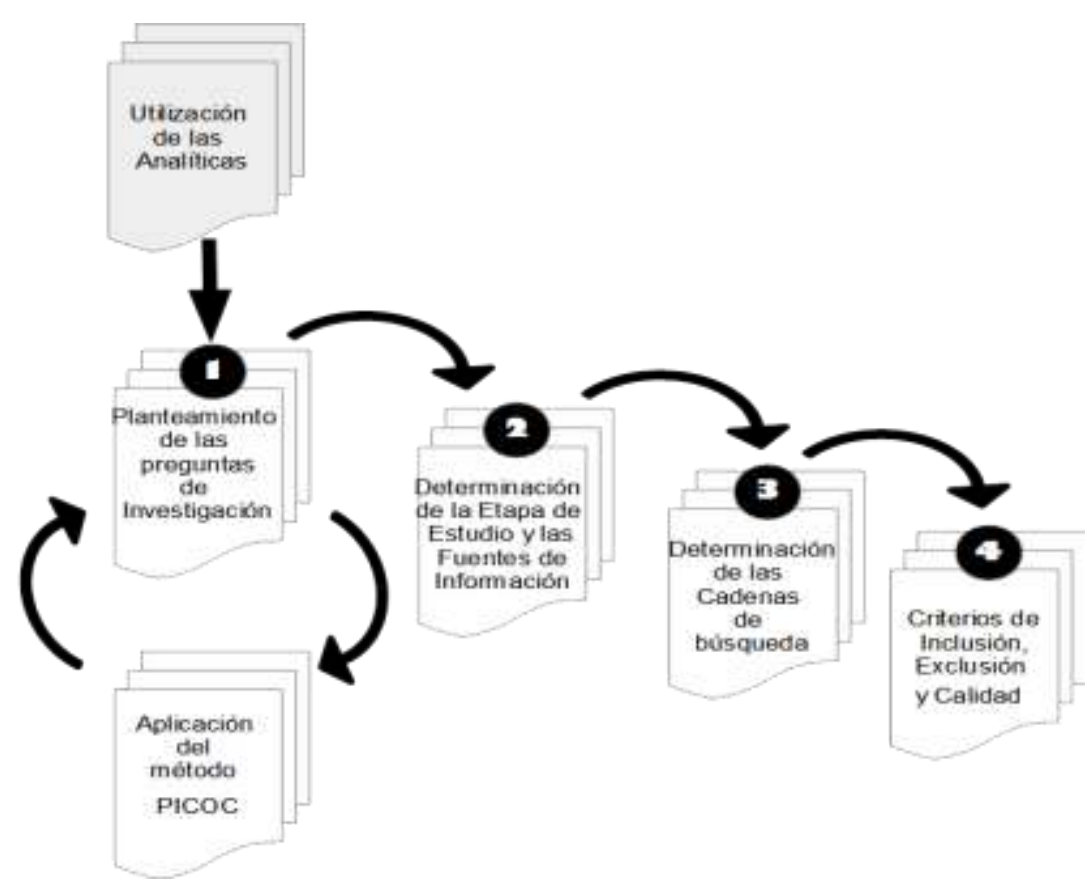

Figura 2. Pasos en el protocolo de Mapeo Sistemático.

Fuente: Elaboración propia.

\subsection{Planteamiento de las Preguntas de Investigación}

El Mapeo Sistemático de Literatura permitió responder el siguiente listado de preguntas de investigación que se encuentra en la Tabla 1:

Tabla 1. Listado de preguntas de investigación.

\begin{tabular}{ll}
\hline FORMA DE & PREGUNTA DE INVESTIGACION (EN EL \\
APLICACIÓN DE LAS & CONTEXTO DE LAS ANALÍTICAS) \\
ANALÍTICAS DE & \\
APRENDIZAJE & \\
\hline PI1, DESCRIPTIVA & $\begin{array}{l}\text { ¿Qué pasó con el total de Trabajos de Titulación } \\
\text { en la ESPOCH por Facultad y por Año? }\end{array}$ \\
\hline PI2, DESCRIPTIVA & $\begin{array}{l}\text { ¿Qué pasó con el total de Trabajos de Titulación } \\
\text { encontrados en la ESPOCH por Carrera y por }\end{array}$ \\
& Año? \\
\hline PI3, DESCRIPTIVA & ¿Qué pasó con los temas clave de los Trabajos \\
& de Titulación por Carrera?
\end{tabular}




\section{PI4, PREDICTIVA ¿Qué pasará con el número de Trabajos de \\ Titulación para el año 2018 y 2019 en la Escuela \\ Superior Politécnica de Chimborazo?}

Fuente: Elaboración propia.

\subsection{Aplicación del método PICOC.}

Petticrew y Roberts (Petticrew \& Roberts, 2008), plantearon el método PICOC de la Tabla 2, para precisar el alcance en la investigación.

Tabla 2. Método PICOC.

\begin{tabular}{ll}
\hline PICOC & SIGNIFICADO \\
\hline Población (P) & $\begin{array}{l}\text { Trabajos de Titulación publicados en la Escuela } \\
\text { Superior Politécnica de Chimborazo }\end{array}$ \\
\hline Intervención (I) & No aplica. \\
\hline Comparación (C) & No aplica. \\
\hline Resultados (O) & Trabajos de Titulación en el período 2010-2017, \\
publicados en la Escuela Superior Politécnica de \\
Chimborazo
\end{tabular}

Fuente: Elaboración propia.

- Determinación de la Etapa de estudio

Se abarcó el mayor número de Trabajos de Titulación, es por ello que se consideraron desde el año 2010 hasta el año 2017, es decir en los últimos 8 años.

\section{- Determinación de las Fuentes de información}

En la Tabla 3, se muestran las fuentes de información y el enlace de acceso:

Tabla 3. Fuentes de Información y Enlace.

\begin{tabular}{lll}
\hline FUENTES DE INFORMACIÓN & ENLACE DE ACCESO \\
\hline $\begin{array}{l}\text { Buscador especializado Google } \\
\text { Académico }\end{array}$ & ("Google Académico," 2017:22:57) \\
\hline
\end{tabular}




\begin{tabular}{ll}
\hline La Red CEDIA & ("Repositorio Institucional del Consorcio \\
& Ecuatoriano para el Desarrollo de \\
& Internet Avanzado," 2018:39:58) \\
\hline Repositorio DSpace & ("DSpace User Registry | DuraSpace," \\
& $2017: 00: 26)$ \\
\hline
\end{tabular}

Fuente: Elaboración propia.

\section{- Determinación de las Cadenas de búsqueda}

Las cadenas de búsqueda están en dependencia de la fuente: Para el repositorio DSpace de la ESPOCH primeramente se seleccionó como comunidad la Facultad, luego las colecciones fueron las Escuelas o Carreras y finalmente se aplicó el buscador compuesto en el campo Fecha de Publicación: 2010, 2011, 2012, 2013, 2014, 2015, 2016, 2017; para delimitar los Trabajos de Titulación por años. Para el caso de la Red CEDIA se utilizó la búsqueda avanzada, en el campo Limitar se ingresó la Escuela Superior Politécnica de Chimborazo, en el campo Formato se colocó Tesis de grado y en el campo lenguaje se ingresó español, además en Año de Publicación se utilizaron los deslizadores para en la opción De ubicar 2010 y en la opción a ubicar 2017. En el buscador especializado google académico se ingresó en la barra de búsquedas simples el título del trabajo de titulación.

\subsection{Criterios de inclusión y exclusión}

En la Tabla 4 se muestran los criterios de inclusión y en la Tabla 5 los criterios de exclusión (Kitchenham et al., 2010)

Tabla 4. Listado de los criterios de inclusión

\begin{tabular}{cl}
\hline NÚMERO & CRITERIOS DE INCLUSION \\
\hline 1 & Si son Trabajos de Titulación de la Escuela Superior \\
& Politécnica de Chimborazo \\
\hline 2 & Si los Trabajos de Titulación no hacen parte de la literatura \\
& gris \\
\hline 3 & Si los Trabajos de Titulación son generados por bases de \\
& datos bibliográficas especializadas \\
\hline 4 & Si los Trabajos de Titulación se encuentran en la etapa \\
& definida en la planificación del Mapeo Sistemático
\end{tabular}

Fuente: Elaboración propia. 
Tabla 5. Listado de los criterios de exclusión

\begin{tabular}{cl}
\hline NÚMERO & CRITERIOS DE EXCLUSION \\
\hline 1 & Si no cumplen uno de los criterios de inclusión \\
\hline 2 & Si existen Trabajos de Titulación duplicados \\
\hline 3 & Si no se puede tener acceso al documento digital completo \\
\hline 4 & Si el Trabajo de Titulación no está escrito en español \\
\hline 5 & $\begin{array}{l}\text { Si los datos de las fuentes no coinciden con el archivo en } \\
\text { formato pdf }\end{array}$
\end{tabular}

Fuente: Elaboración propia.

\subsection{Criterios de Calidad}

Los artículos científicos tuvieron que cumplir los criterios de calidad dados por las respuestas a las preguntas de la Tabla 6:

Tabla 6. Criterios de calidad.

\begin{tabular}{cl}
\hline NÚMERO & CRITERIOS DE CALIDAD \\
\hline 1 & $\begin{array}{l}\text { ¿Los Trabajos de Titulación fueron extraídos de una fuente } \\
\text { confiable? }\end{array}$ \\
\hline 2 & $\begin{array}{c}\text { ¿Los Trabajos de Titulación están respaldados digitalmente } \\
\text { por la entidad legal? }\end{array}$ \\
\hline 4 & ¿Los Trabajos de Titulación están digitalizados \\
& completamente? \\
\hline 5 & $\begin{array}{l}\text { ¿Los Trabajos de Titulación tienen el oficial de la entidad } \\
\text { competente? }\end{array}$
\end{tabular}

Fuente: Elaboración propia.

\section{RESULTADOS Y DISCUSIÓN}

Todos los resultados que se exponen a continuación han sido extraídos de las fuentes de información definidas en los pasos del protocolo de Mapeo Sistemático actualizados a la fecha 24 de agosto del 2018. 


\section{PI1, DESCRIPTIVA: ¿Qué pasó con el total de Trabajos de Titulación en la ESPOCH por} Facultad y por Año?

Los Trabajos de Titulación son registrados en la ESPOCH desde el año 2009, los técnicos por facultad de la biblioteca son los encargados en ingresar la información de los Trabajos de Titulación. La Tabla 7, muestra el total de Trabajos de Titulación por Año y por cada una de las 7 Facultades de la ESPOCH, la tabla contiene aproximadamente un $97 \%$ de los Trabajos de Titulación (7097), el porcentaje faltante $3 \%$ pertenece a los trabajos de graduación del año 2009 y a los del año 2018.

Tabla 7. Número de Trabajos de Titulación en los últimos 8 años por Facultad en la ESPOCH.

\begin{tabular}{|c|c|c|c|c|c|c|c|c|c|}
\hline \multirow{2}{*}{ FACULTAD } & \multicolumn{9}{|c|}{ AÑOS } \\
\hline & 2010 & 2011 & 2012 & 2013 & 2014 & 2015 & 2016 & 2017 & TOTAL \\
\hline CIENCIAS & 58 & 51 & 135 & 141 & 156 & 291 & 202 & 210 & 124494,84 \\
\hline \multicolumn{10}{|l|}{ INFORMÁTICA Y } \\
\hline ELECTRÓNICA & 81 & 70 & 112 & 135 & 175 & 152 & 197 & 151 & 107399,34 \\
\hline RECURSOS NATURALES & 43 & 177 & 90 & 79 & 73 & 118 & 123 & 99 & 80294,2 \\
\hline \multicolumn{10}{|l|}{ ADMINISTRACION DE } \\
\hline EMPRESAS & 42 & 59 & 102 & 160 & 191 & 287 & 304 & 257 & 140296,37 \\
\hline CIENCIAS PECUARIAS & 4 & 176 & 279 & 102 & 41 & 185 & 127 & 113 & 102797,86 \\
\hline MECÁNICA & 96 & 108 & 107 & 137 & 222 & 107 & 132 & 166 & 107599,13 \\
\hline SALUD PÚBLICA & 21 & 58 & 91 & 84 & 111 & 98 & 9 & 2 & $474 \quad 97,68$ \\
\hline TOTAL & 345 & 699 & 916 & 838 & 969 & 1238 & 1094 & 998 & 709797,06 \\
\hline
\end{tabular}

Fuente: Elaboración propia.

En la gráfica de barras de la Figura 3, al igual que en la tabla anterior se puede observar que las Facultades de Administración de Empresas, Ciencias y Ciencias Pecuarias son las que tienen mayor número de Trabajos de Titulación tienen registradas en dependencia del año de referencia. Sobresale en el año 2012 el número de Trabajos de Titulación de Ciencias Pecuarias.

Tabla 8. Número total de Trabajos de Titulación por Escuela y por Año.

\begin{tabular}{lllllllllll}
\hline & \multicolumn{11}{c}{ AÑOS } \\
\cline { 3 - 8 } FACULTAD & CARRERAS & 2 & 2 & 2 & 2 & 2 & 2 & 2 & 2 & T \\
& CARE & 0 & 0 & 0 & 0 & 0 & 0 & 0 & 0 & 0 \\
\hline
\end{tabular}




\begin{tabular}{|c|c|c|c|c|c|c|c|c|c|c|}
\hline & & 1 & 1 & 1 & 1 & 1 & 1 & 1 & 1 & $\mathbf{T}$ \\
\hline & & 7 & 6 & 5 & 4 & 3 & 2 & 1 & 0 & \\
\hline & INGENIERÍA QUÍMICA & 54 & 42 & 66 & 5 & 3 & 35 & 8 & 1 & 308 \\
\hline & & & & & 0 & 8 & & & 5 & \\
\hline & $\begin{array}{l}\text { ESTADISTICA INFORMATICA } \\
\end{array}$ & 1 & 4 & 3 & 4 & 5 & 16 & 1 & 0 & 34 \\
\hline & MATEMATICA & 0 & 0 & 0 & 0 & 0 & 0 & 0 & 0 & 0 \\
\hline & FISICA & 0 & 0 & 0 & 0 & 0 & 0 & 0 & 0 & 0 \\
\hline CIENCIAS & BIOFISICA & 10 & 9 & 11 & 5 & 4 & 7 & 0 & 0 & 46 \\
\hline & ESTADISTICA & 0 & 0 & 0 & 0 & 0 & 0 & 0 & 0 & 0 \\
\hline & QUIMICA & 0 & 0 & 0 & 0 & 0 & 0 & 0 & 0 & 0 \\
\hline & BIOTECNOLOGIA AMBIENTAL & 77 & 73 & 70 & 2 & 1 & 24 & 4 & 1 & 307 \\
\hline & & & & & 9 & 1 & & & 9 & \\
\hline & $\overline{\text { BIOQUIMICA Y FARMACIA }}$ & 68 & 74 & 14 & 6 & 8 & 53 & 3 & 2 & 549 \\
\hline & & & & 1 & 8 & 3 & & 8 & 4 & \\
\hline & DISEÑO GRÁFICO & 42 & 56 & 44 & 6 & 3 & 39 & 2 & 1 & 330 \\
\hline & & & & & 8 & 6 & & 6 & 9 & \\
\hline & INGENIERİA EN ELECTRÓNICA, & 27 & 41 & 33 & 3 & 4 & 11 & 1 & 1 & 209 \\
\hline & CONTROL Y REDES & & & & 9 & 3 & & 4 & & \\
\hline INFORMÁTIC & INDUSTRIALES & & & & & & & & & \\
\hline$A Y$ & ELECTRÓNICA Y & 0 & 0 & 0 & 0 & 0 & 0 & 0 & 0 & 0 \\
\hline ELECTRÓNIC & AUTOMATIZACIÓN & & & & & & & & & \\
\hline A & INGENIERÍA EN ELECTRÓNICA, & 53 & 45 & 30 & 2 & 1 & 9 & 8 & 1 & 186 \\
\hline & TELECOMUNICACIONES Y & & & & 3 & 7 & & & & \\
\hline & REDES & & & & & & & & & \\
\hline & TELECOMUNICACIONES & 0 & 0 & 0 & 0 & 0 & 0 & 0 & 0 & 0 \\
\hline & INGENIERÍA EN SISTEMAS & 29 & 55 & 45 & 4 & 3 & 53 & 2 & 6 & 348 \\
\hline & & & & & 5 & 9 & & 2 & 0 & \\
\hline & SOFTWARE & 0 & 0 & 0 & 0 & 0 & 0 & 0 & 0 & 0 \\
\hline & AGRONOMÍA & 15 & 19 & 23 & 2 & 2 & 36 & 5 & 4 & 234 \\
\hline & & & & & 0 & 1 & & 8 & 2 & \\
\hline RECURSOS & TURISMO SOSTENIBLE & 10 & 10 & 3 & 0 & 0 & 0 & 0 & 0 & 23 \\
\hline NATURALES & ECOTURISMO & 59 & 82 & 75 & 4 & 4 & 35 & 9 & 1 & 434 \\
\hline & & & & & 5 & 4 & & 3 & & \\
\hline & INGENIERÍA FORESTAL & 15 & 12 & 17 & 8 & 1 & 19 & 2 & 0 & 111 \\
\hline & & & & & & 4 & & 6 & & \\
\hline
\end{tabular}




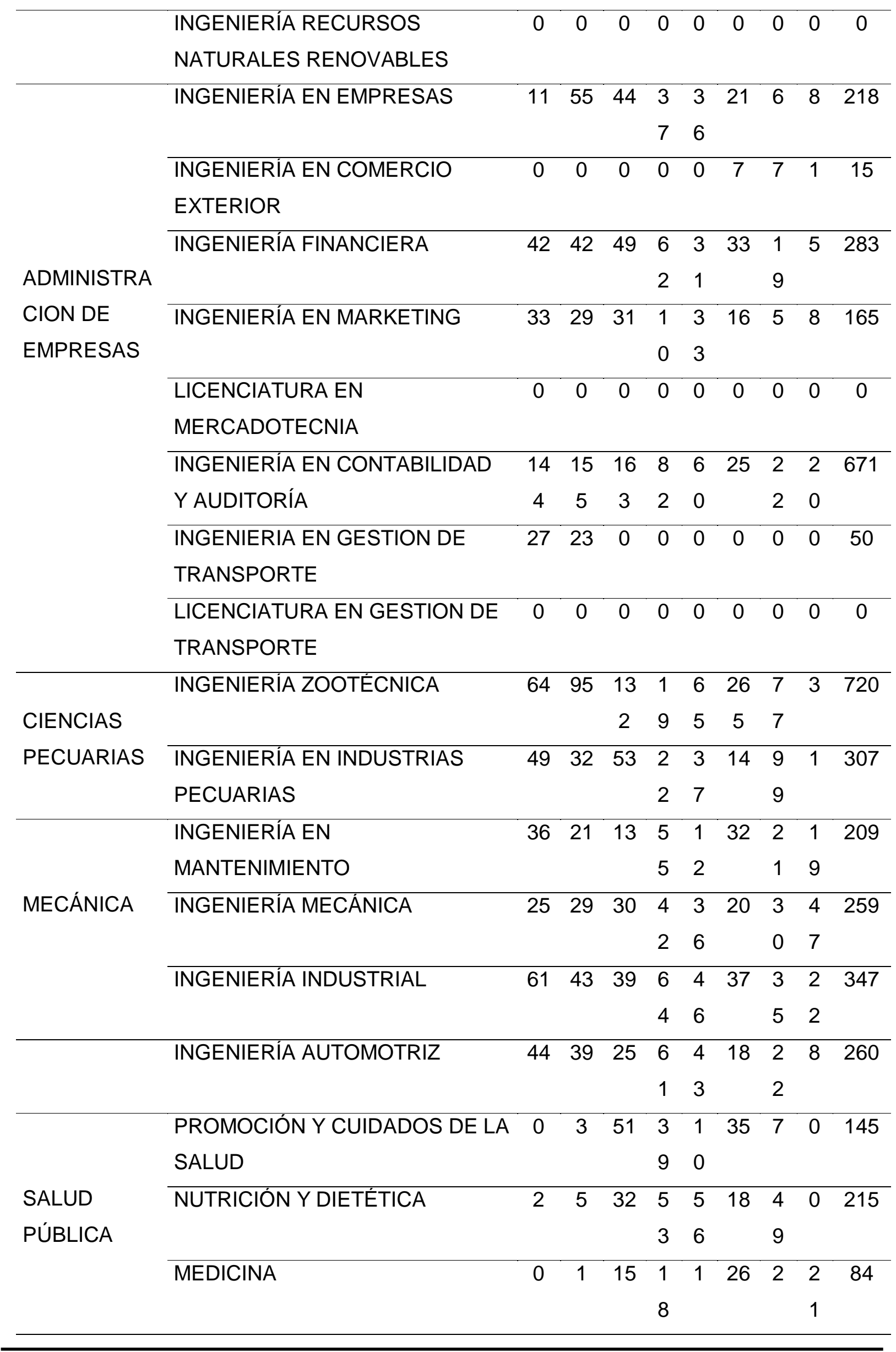




\section{GASTRONOMÍA

$\begin{array}{lllllllll}0 & 0 & 0 & 1 & 1 & 12 & 0 & 0 & 30\end{array}$ \\ 7}

Fuente: Elaboración propia.

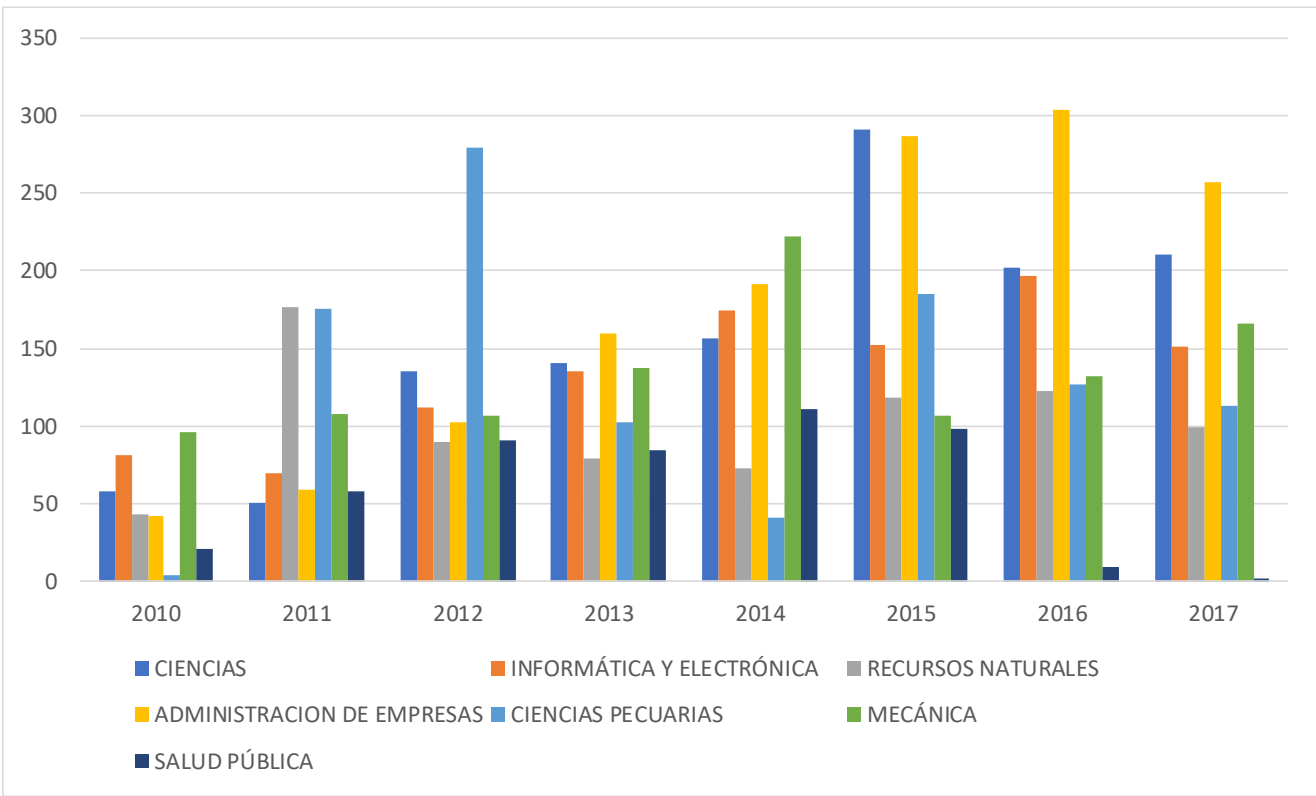

Figura 3. Gráfico de Barras comparativo por años entre las 7 Facultades de la ESPOCH. Fuente: Elaboración propia.

En general la mayor producción de Trabajos de Titulación se encuentra en la Facultad de Administración de Empresas, seguido por la Facultad de Ciencias y la Facultad de Informática y Electrónica. La Facultad con un menor número de Trabajos de Titulación es la de Salud Pública.

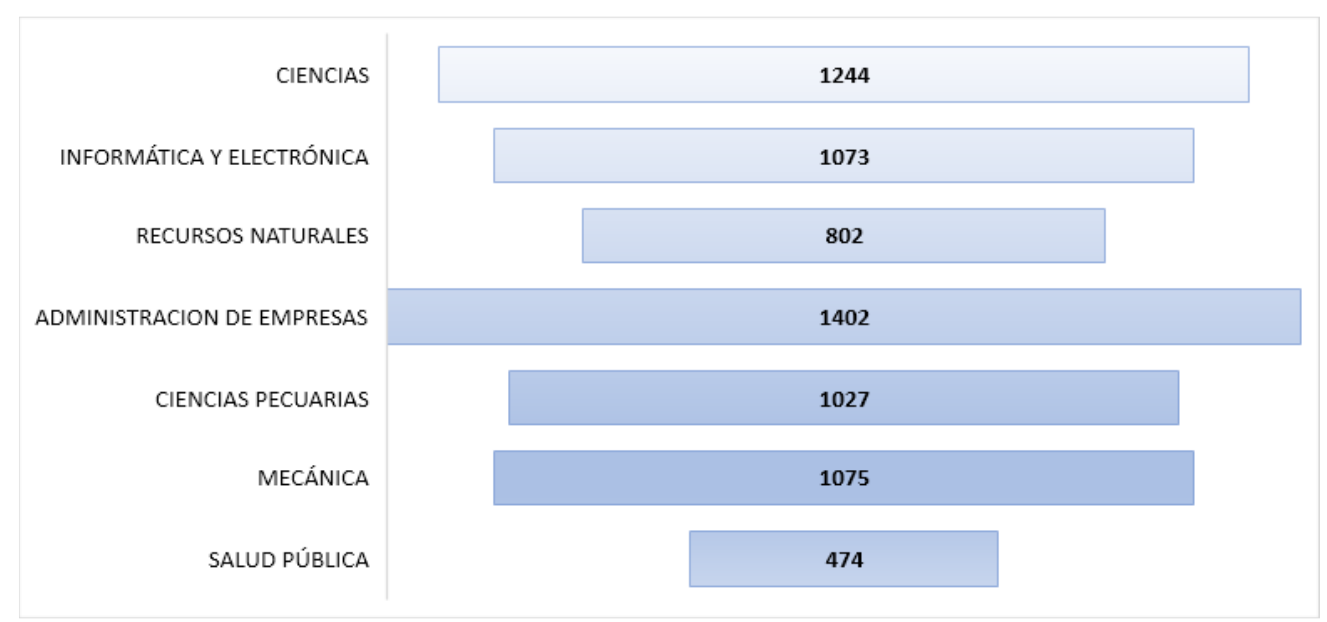

Figura 4. Gráfico comparativo del número total de Trabajos de Titulación entre las Facultades de la ESPOCH en los últimos 8 años.

Fuente: Elaboración propia. 
PI2, DESCRIPTIVA: ¿Qué pasó con el total de Trabajos de Titulación encontrados en la ESPOCH por Carrera y por Año?

La ESPOCH, tiene una estructura jerárquica de Facultades, Escuelas y Carreras, la tabla 8 muestra en detalle el número de Trabajos de Titulación por Facultad, Carrera y años en forma descendente desde el 2017 hasta el 2010. Las Carreras que muestran valores de cero en sus celdas significa que son reformulación de otras carreras o carreras nuevas.

La Figura 5, muestra el gráfico de barras comparativo para las 39 carreras de la ESPOCH, observándose que la carrera de Ingeniería en Zootecnia tiene el número más alto de Trabajos de Titulación seguidos por las carreras de Ingeniería en Contabilidad y auditoría y la Carrera de Bioquímica y Farmacia, perteneciente a la Facultad de Ciencias. La carrera de Ingeniería en Comercio exterior es la que menos Trabajos de Titulación posee siendo únicamente 15, seguido de Turismo sostenible con 23 y Gastronomía con 30.

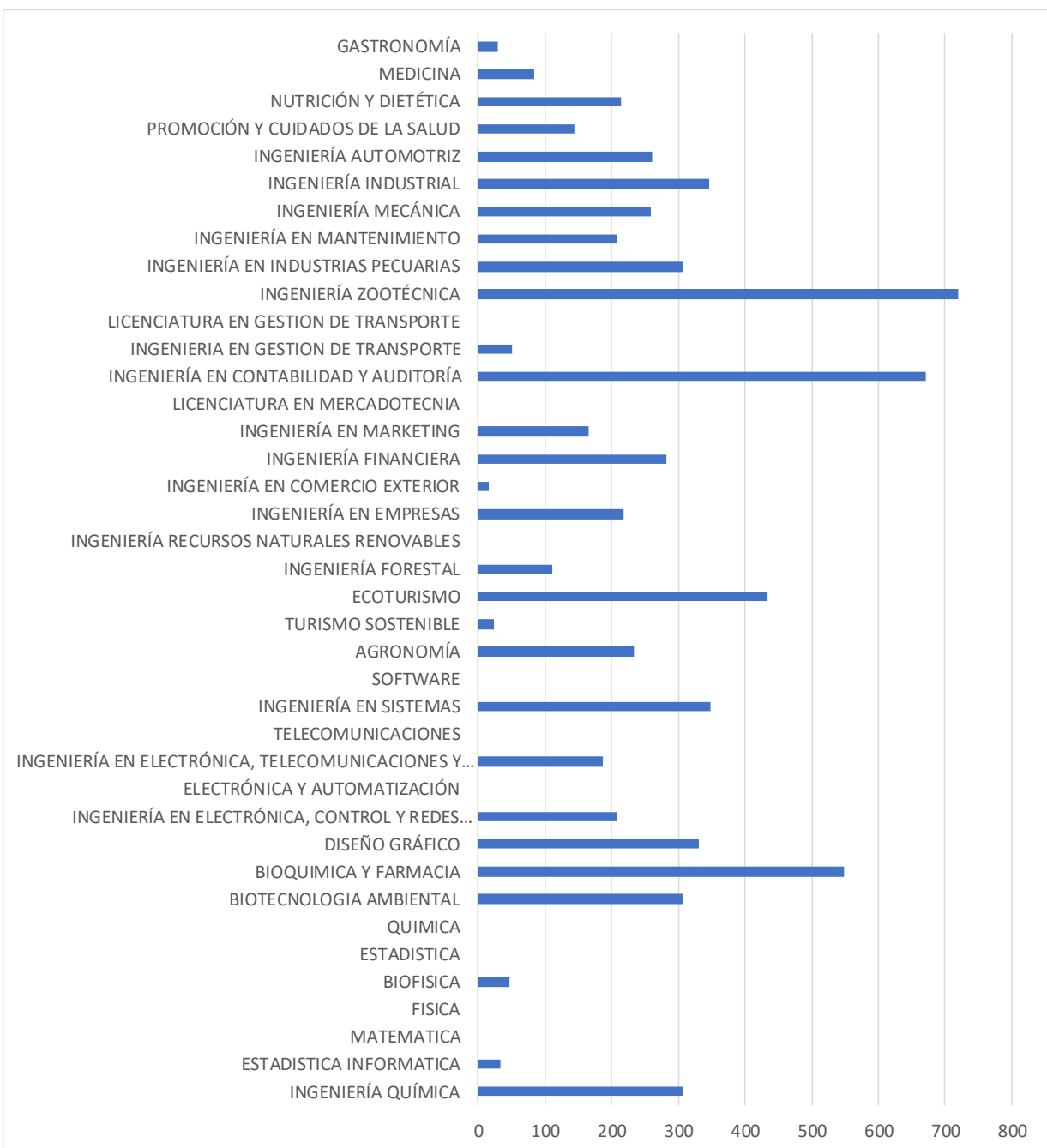

Figura 5: Gráfico de barras horizontal del total de Trabajos de Titulación en los últimos 8 años.

Fuente: Elaboración propia. 
PI3, DESCRIPTIVA: ¿Qué pasó con los temas clave de los Trabajos de Titulación por Carrera?

Los temas de los Trabajos de Titulación son un indicativo importante de las temáticas de la Investigación de Pregrado que se lleva en las Instituciones de Educación Superior, los temas clave son ingresados por especialistas bibliotecarios al almacenar y digitalizar la información de los trabajos de graduación.

Para cada una de las carreras, la Tabla 9 muestra los temas con su frecuencia absoluta en orden decreciente por fila. Por la extensión de la tabla no se la muestra toda la información, pero se puede acceder a la información completa mediante el enlace: https://drive.google.com/file/d/1c4-Q-qnU8-mv9Y S-cqT1mA7pBx9xcsP/view?usp=sharing

Tabla 9. Temas clave de los Trabajos de Titulación por Carrera.

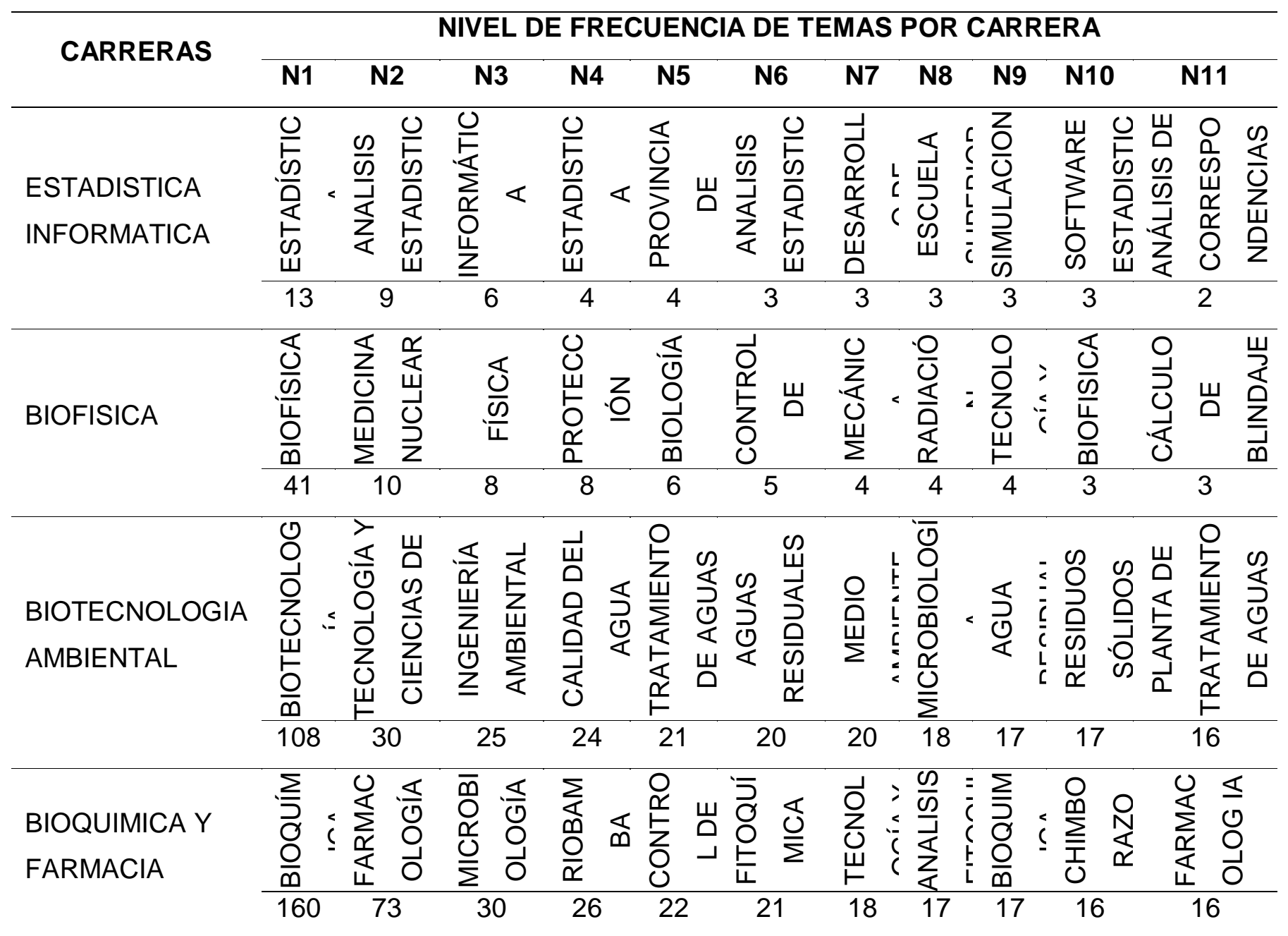

Fuente: Elaboración propia. 
En el $80 \%$ de la tabla 9, el nombre de la carrera coincide con el tema clave de mayor frecuencia, esto es un indicativo de que la mayoría de los temas de los Trabajos de Titulación guardan estrecha relación con la Carrera de la cual se originan.

\section{PI4, PREDICTIVA: ¿Qué pasará con el número de Trabajos de Titulación para el año 2018 y 2019 en la Escuela Superior Politécnica de Chimborazo?}

A continuación, se utiliza la Analítica Predictiva y en particular las medias móviles no ponderadas de tres términos para calcular las tendencias en los próximos 2 años y con ellas realizar una aproximación al total de Trabajos de Titulación y la cantidad de material de estudio que se tendrá para estudiar el estado de la Investigación de pregrado en la ESPOCH. La figura 9, muestra la gráfica de líneas de la serie temporal del total Trabajos de Titulación en los años del 2010 al 2017 y la gráfica de pronósticos con promedios móviles de tres períodos.

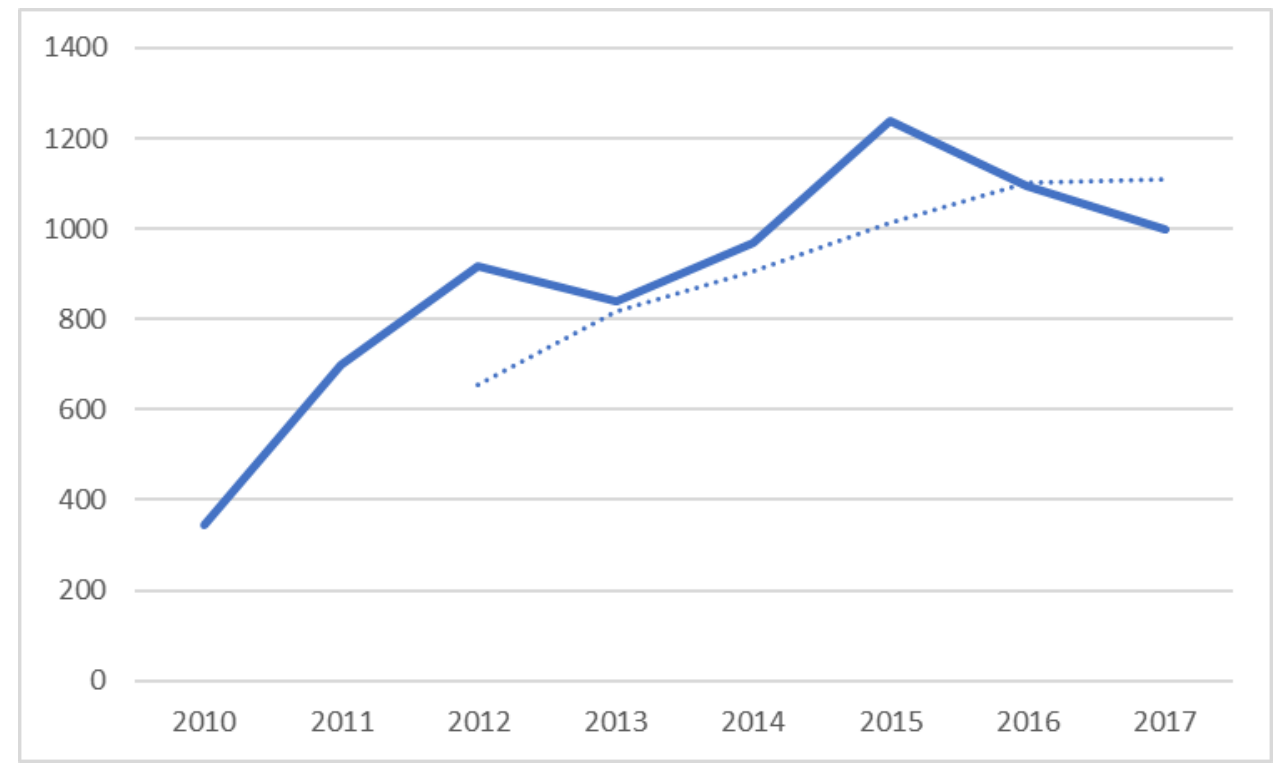

Figura 9: Gráfico de Líneas y de pronósticos con promedios móviles de tres períodos.

La tabla 10, muestra los resultados del cálculo de tendencia con Media Móvil de tres términos (años) aplicados a las 7 facultades y al total de Trabajos de Titulación de la ESPOCH. Se puede observar por ejemplo que la Facultad de Ciencias tiene una proyección de 234 Trabajos de Titulación para el 2018, 228 para el 2019 y un total proyectado de 1073 Trabajos de Titulación desde el 2010 hasta el 2019 conociendo que hasta el 2017 se tenía un total de 1214 Trabajos de Titulación. 
Tabla 10. Tendencia en el 2018, 2019 y total de trabajos para la ESPOCH y sus Facultades.

\begin{tabular}{|c|c|c|c|c|c|c|c|c|c|c|c|c|c|c|c|c|}
\hline \multirow[t]{2}{*}{$\begin{array}{l}\text { AÑOS } \\
2010\end{array}$} & \multicolumn{2}{|c|}{$\frac{0}{\frac{0}{0}}$} & \multicolumn{2}{|c|}{ 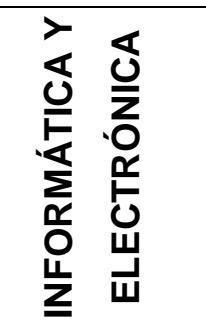 } & \multirow[t]{2}{*}{ 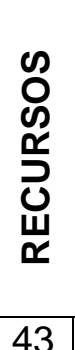 } & 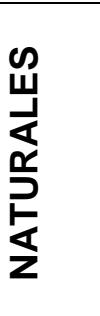 & \multirow[t]{2}{*}{ 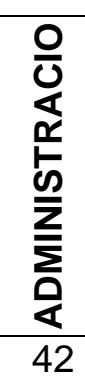 } & \multirow[t]{2}{*}{ 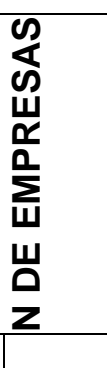 } & $\begin{array}{l}0 \\
\frac{0}{0} \\
\frac{U}{0}\end{array}$ & 先 & \multicolumn{2}{|c|}{ 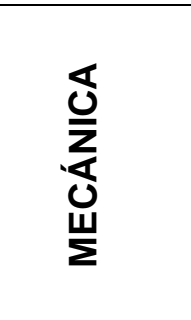 } & \multicolumn{2}{|c|}{ 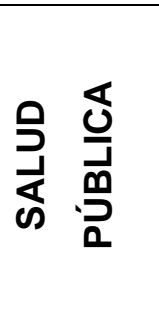 } & \multicolumn{2}{|c|}{$\begin{array}{l}\text { T } \\
0 \\
0 \\
0 \\
0 \\
\square\end{array}$} \\
\hline & 58 & & 81 & & & & & & 4 & & 96 & & 21 & & 345 & \\
\hline 2011 & 51 & & 70 & & $\begin{array}{r}17 \\
7\end{array}$ & & 59 & & 176 & & 108 & & 58 & & 699 & \\
\hline 2012 & 135 & 81 & 112 & 88 & 90 & 103 & 102 & 68 & 279 & 153 & 107 & 104 & 91 & 57 & 916 & $\begin{array}{r}65 \\
3\end{array}$ \\
\hline 2013 & 141 & 109 & 135 & 106 & 79 & 115 & 160 & 107 & 102 & 186 & 137 & 117 & 84 & 78 & 838 & $\begin{array}{r}81 \\
8\end{array}$ \\
\hline 2014 & 156 & 144 & 175 & 141 & 73 & 81 & 191 & 151 & 41 & 141 & 222 & 155 & $\begin{array}{r}11 \\
1\end{array}$ & 95 & 969 & $\begin{array}{r}90 \\
8\end{array}$ \\
\hline 2015 & 291 & 196 & 152 & 154 & $\begin{array}{r}11 \\
8\end{array}$ & 90 & 287 & 213 & 185 & 109 & 107 & 155 & 98 & 98 & $\begin{array}{r}123 \\
8\end{array}$ & $\begin{array}{l}10 \\
15\end{array}$ \\
\hline 2016 & 202 & 216 & 197 & 175 & $\begin{array}{r}12 \\
3\end{array}$ & 105 & 304 & 261 & 127 & 118 & 132 & 154 & 9 & 73 & $\begin{array}{r}109 \\
4\end{array}$ & $\begin{array}{l}11 \\
00\end{array}$ \\
\hline 2017 & 210 & 234 & 151 & 167 & 99 & 113 & 257 & 283 & 113 & 142 & 166 & 135 & 2 & 36 & 998 & $\begin{array}{l}11 \\
10\end{array}$ \\
\hline $\begin{array}{l}\text { Proytd } \\
2018\end{array}$ & & 234 & & 167 & & 113 & & 283 & & 142 & & 135 & & 36 & & $\begin{array}{l}11 \\
10\end{array}$ \\
\hline $\begin{array}{l}\text { Proytd } \\
2019\end{array}$ & & 228 & & 170 & & 110 & & 276 & & 134 & & 141 & & 48 & & $\begin{array}{l}11 \\
07\end{array}$ \\
\hline TOTAL & 124 & & 107 & & 80 & & 140 & & 102 & & 107 & & 47 & & 709 & \\
\hline 8 años & 4 & & 3 & & 2 & & 2 & & 7 & & 5 & & 4 & & 7 & \\
\hline $\begin{array}{l}\text { Total } \\
\text { Proytd } \\
10 \\
\text { años }\end{array}$ & & $\begin{array}{r}170 \\
6\end{array}$ & & $\begin{array}{r}141 \\
0\end{array}$ & & $\begin{array}{r}102 \\
5\end{array}$ & & $\begin{array}{r}196 \\
1\end{array}$ & & $\begin{array}{r}130 \\
3\end{array}$ & & $\begin{array}{r}135 \\
1\end{array}$ & & $\begin{array}{r}55 \\
8\end{array}$ & & $\begin{array}{l}93 \\
14\end{array}$ \\
\hline
\end{tabular}

Fuente: Elaboración propia. 
Se recomienda complementar este estudio predictivo con el cálculo de errores y usando otras técnicas Predictivas como Las medias ponderadas de tres términos.

\section{CONCLUSIONES}

Con este trabajo se realizó un estudio de la Investigación de Pregrado en la Escuela Superior Politécnica de Chimborazo, desde el contexto de las Analíticas; basándose en los Trabajos de Titulación de los últimos 8 años, que se encuentran alojados en las fuentes oficiales institucionales. Se utilizó el Mapeo Sistemático de literatura, para revisar el 97,06\% de Trabajos de Titulación, correspondientes a 7097 de los 7314 totales. Todos los resultados han sido extraídos de las fuentes definidas en el Mapeo Sistemático, además se aplicaron las Analíticas de Aprendizaje Descriptivas a las tres primeras preguntas y Analíticas Predictivas en la cuarta pregunta.

Las Analíticas de Aprendizaje Descriptivas permitieron responder a las preguntas ¿Qué pasó con el total de Trabajos de Titulación en la ESPOCH por Facultad y por Año? y ¿Qué pasó con el total de Trabajos de Titulación encontrados en la ESPOCH por Carrera y por Año? y de esta forma establecer el estado actual y caracterizar la Investigación de Pregrado en la ESPOCH, para ello se utilizaron tablas de frecuencia y gráficos de frecuencia absoluta y porcentual. En general tanto las Facultades de Ciencias, así como la de Administración de empresas son las que tienen un mayor número de c en todos los años, llama la atención que en el año 2012 el número de Trabajos de Titulación de Ciencias Pecuarias es el más alto que el resto de Las facultades. La mayor producción de Trabajos de Titulación se encuentra en la Facultad de Administración de Empresas y la menor en la Facultad de Salud Pública. Existen 10 Carreras que muestran valores de cero en sus celdas significando que son reformulación de otras carreras (Estadística por Estadística Informática, Química por Ingeniería Química, Licenciatura en Gestión de Transporte por Ingeniería en Gestión de Transporte) o carreras nuevas (Matemática, Física). La carrera de Ingeniería en Zootecnia tiene el número más alto y la carrera de Ingeniería en Comercio exterior tiene el número más bajo de Trabajos de Titulación.

Las Analíticas de Aprendizaje Descriptivas permitieron responder a la pregunta tres ¿Qué pasó con los temas clave de los Trabajos de Titulación por Carrera? Los temas clave de los Trabajos de Titulación son un indicativo importante de las temáticas y algunas características importantes de la Investigación de Pregrado, se observa que la mayoría de los temas clave de los Trabajos de Titulación guardan directa relación con la Carrera de la cual se originan. 
En la cuarta pregunta de investigación se aplicó las Analíticas de Aprendizaje Proyectivas permitieron aproximarnos al futuro de la investigación de pregrado en la ESPOCH respondiendo a la pregunta ¿qué pasará con la cantidad de Trabajos de Titulación en la ESPOCH? y para contestarla se utilizó la técnica de medias móviles para series temporales. Además, se proyectó proyectar el número de Trabajos de Titulación en la ESPOCH en los años 2018, 2019 y el total después de 10 años, obteniéndose 1110, 1107 y 9314 respectivamente.

Una de las limitaciones de este trabajo fue su directa dependencia a la exactitud y precisión de los datos almacenados en las fuentes de información utilizadas, pero una de sus fortalezas fue la independencia en el tratado de las fuentes primarias. Esta investigación es totalmente replicable a cualquier Institución de Educación Superior Ecuatoriana e Internacional si utiliza fuentes similares de información. Se sugiere que como siguiente paso se utilicen las Analíticas de Diagnóstico y las Analíticas Prescriptiva en los ámbitos de interés de estudiantes, docentes, investigadores y sobre todo de los gestores y encargados de la toma de decisiones en el área de Investigación, debido a que los resultados obtenidos se enmarcan y serán de especial beneficio en las Analíticas Académicas.

\section{AGRADECIMIENTOS}

A la Escuela Superior Politécnica de Chimborazo que mediante el apoyo al proyecto "Análisis e innovación de las investigaciones en la Facultad de Ciencias como estrategia para incrementar la producción científica", permite que se analice, mejore e incremente la investigación en la Facultad de Ciencias y en la ESPOCH. A los investigadores del grupo de investigación en Ciencia de Datos - CIDED (CIDED, 2017). A Gabriela Ashqui Salazar por su ayuda en la tabulación de datos.

\section{REFERENCIAS BIBLIOGRÁFICAS}

1st International Conference on Learning Analytics and Knowledge 2011 | Connecting the technical, pedagogical, and social dimensions of learning analytics. (2018:54:26).

Retrieved from https://tekri.athabascau.ca/analytics/

CIDED. (2017). Inicio - Grupo de Investigación Ciencia de Datos-CIDED.

Deitz-Uhler, B. \& Hurn, J. Using Learning Analytics to Predict (and Improve) Student

Success: A Faculty Perspective.

Drupal. (2011). 1st International Conference on Learning analytics and Knowledge 2011.

DSpace User Registry | DuraSpace. (2017:00:26). 
Espoch. (2018). Inicio - Escuela Superior Politécnica de Chimborazo.

Facultad de Administración de Empresas - Escuela Superior Politécnica de Chimborazo. (2018:19:07). Retrieved from https://www.espoch.edu.ec/index.php/facultades.html?id=193

Facultad de Ciencias - Escuela Superior Politécnica de Chimborazo. (2018:46:41). Retrieved from https://www.espoch.edu.ec/index.php/component/k2/item/1313.html

Facultad de Ciencias Pecuarias - Escuela Superior Politécnica de Chimborazo. (2018:32:28). Retrieved from https://www.espoch.edu.ec/index.php/facultades.html?id=196

Facultad de Informática y Electrónica - Escuela Superior Politécnica de Chimborazo. (2018:28:23). Retrieved from https://www.espoch.edu.ec/index.php/facultades.html?id=197

Facultad de Mecánica - Escuela Superior Politécnica de Chimborazo. (2018:35:35). Retrieved from https://www.espoch.edu.ec/index.php/facultades.html?id=198

Facultad de Recursos Naturales - Escuela Superior Politécnica de Chimborazo. (2018:38:44). Retrieved from https://www.espoch.edu.ec/index.php/facultad-de-recursosnaturales.html

Facultad de Salud Pública - Escuela Superior Politécnica de Chimborazo. (2018:44:46). Retrieved from https://www.espoch.edu.ec/index.php/facultades.html?id=199

Fidalgo-Blanco, Á., Sein-Echaluce, M. L., García-Peñalvo, F. J., \& Conde, M. Á. (2015). Using Learning Analytics to improve teamwork assessment. Computers in Human Behavior, 47, 149-156. https://doi.org/10.1016/j.chb.2014.11.050

Gartner. (2018:36:38). Retrieved from https://www.gartner.com/en Gelan, A., Fastré, G., Verjans, M., Martin, N., Janssenswillen, G., Creemers, M., . . . Thomas, M. (2018). Affordances and limitations of learning analytics for computerassisted language learning: A case study of the VITAL project. Computer Assisted Language Learning, 31(3), 294-319. https://doi.org/10.1080/09588221.2017.1418382 Google Académico. (2017:22:57).

El Plan Nacional para el Buen Vivir ya está disponible para la ciudadanía | Secretaría Nacional de Planificación y Desarrollo. (n.d.). Retrieved November 19, 2018, from http://www.planificacion.gob.ec/el-plan-nacional-para-el-buen-vivir-ya-esta-disponiblepara-la-ciudadania/

Haro, S.; Pazmiño, R.; Conde, M., \& Peñalvo, F. (2018). Data mining to discover the classification trend in titling works.

IDI. (2014a). PLAN DE INVESTIGACION ESPOCH 2014-2018. Ecuador. 
IDI. (2014b). REGLAMENTO DEL INSTITUTO DE INVESTIGACIONES DE LA ESCUELA SUPERIOR POLITECNICA DE CHIMBORAZO 2014. Ecuador.

IDI. (2018). IDI home page.

Index of /images/Comunicacion/OCTUBRE 2017/COMUNICADOS. (2018:26:09). Retrieved from

https://www.espoch.edu.ec/images/Comunicacion/OCTUBRE\%202017/COMUNICADOS/

Kitchenham, B., Pretorius, R., Budgen, D., Brereton, O. P., Turner, M., Niazi, M., \& Linkman, S. (2010). Systematic literature reviews in software engineering-a tertiary study. Information and Software Technology, 52(8), 792-805.

La Ley Orgánica Reformatoria a Ley Orgánica de Educación Superior fue publica en el Registro Oficial - Ediciones Legales. (s/f). Recuperado el 19 de noviembre de 2018, de http://www.edicioneslegales.com.ec/la-ley-organica-reformatoria-a-ley-organica-deeducacion-superior-fue-publica-en-el-registro-oficial/

LOES. (2010). Ley Orgánica de Educacion Superior. Quito-Ecuador, p. 58.

Manosalvas, M. (05/19/). Buen vivir o sumak kawsay. En busca de nuevos referenciales para la acción pública en Ecuador: Íconos - Revista de Ciencias Sociales; Núm. 49 (2014): Dossier: Economía política y políticas democráticas de comunicación en América LatinaDO - 10.17141/iconos.49.2014.1273.

Naranjo, M., Pazmiño, R., Conde, M. \& Peñalvo, F. (2018). LA \& SIA cluster methods: Computational comparison.

Objetivos-del-Milenio-Balance-2014.

Okoli, C., \& Schabram, K. (2010). A guide to conducting a systematic literature review of information systems research. Sprouts Work. Pap. Inf. Syst, 10, 26.

Pazmiño, R., García, F. J., Coutrier, R., \& Conde, M. Á. (2015). Statistical implicative analysis for educational data sets: 2 analysis with RCHIC.

Pazmiño, R., Peñalvo, F.; Conde, M.; Chiluiza, P., \& Campoverde, D. (2017). La estadística implicativa como gestor del conocimiento en el análisis de la investigación universitaria en la Escuela Superior Politécnica de Chimborazo.

Pazmiño, R., Román, E. G., Martínez, E. Y., \& Páez, L. L. (2018). La investigación en Ciencias Ambientales en la Escuela Superior Politécnica de Chimborazo: Características, semejanzas y dependencias: Características, semejanzas y dependencias. Retrieved from

https://knowledgesociety.usal.es/sites/default/files/evidencias/semejanzas\%20y\%20depen dencias\%20ciencias\%20ambientales5\%20correcci\%C3\%B3n2.pdf

Pazmiño, R. A. (2014). Aproximación al Análisis Estadístico Implicativo desde sus Aplicaciones Educativas. 
Pazmiño, R. A., García, F. J.; Conde, M. A., García, M. J. \& Montoya, J. K.. (2018). El Análisis Estadístico Implicativo y la mejora del aprendizaje en el marco de las Analíticas de Aprendizaje: Un Mapeo Sistemático de Literatura: Un Mapeo Sistemático de Literatura. Retrieved from https://knowledgesociety.usal.es/sites/default/files/evidencias/SIAimproveL6send_0.pdf

Pazmiño, R. A.; García, F. J.; Conde, M. A.; Valverde, P. E. \& Montoya, J. K. (2018). Predicción del Aprendizaje y Learning Analytics: Un Mapeo Sistemático del Análisis Estadístico Implicativo: Un Mapeo Sistemático del Análisis Estadístico Implicativo. Retrieved from https://knowledgesociety.usal.es/sites/default/files/evidencias/SIApredictionL8send.pdf

Pazmiño, R. A., García, F. J., \& Conde, M. A. (2016). Approximation of statistical implicative analysis to learning analytics:a systematic review: a systematic review.

Pazmiño, R. A., García, F. J., \& Conde, M. A. (2017a). Comparing Hierarchical Trees in Statistical Implicative Analysis \& Hierarchical Cluster in Learning Analytics.

Pazmiño, R. A., García, F. J., \& Conde, M. A. (2017b). Mapeo Sistemático en B-Learning: Aplicación a cursos moodle sobre Análisis Estadístico Implicativo.

Pazmiño, R. A., García, F. J., \& Conde, M. A. (2017c). Statistical Implicative Analysis aproximation to KDD and Data Mining: A systematic and mapping review in Knowledge Discovery Database framework.

Petticrew, M., \& Roberts, H. (2008). Systematic reviews in the social sciences: A practical guide: John Wiley \& Sons.

Ramírez, R. (2010). Introducción: Transformar la universidad para transformar la sociedad. Quito, 978-9978-92-893-6, p. 254.

Repositorio Institucional del Consorcio Ecuatoriano para el Desarrollo de Internet Avanzado: Página de inicio. (2018:39:58). Retrieved from http://repositorio.cedia.org.ec/ SENPLADES. (2013). Plan Nacional para el Buen Vivir 2013-2017. Quito-Ecuador, ISBN 978-9942-07-463-8, p. 130.

UNESCO. (2018:30:04). Inicio-UNESCO.

UNESCO. General Conference; 36th; Revisión de la Clasificación Internacional Normalizada de la Educación (CINE); 2011. 